\title{
Shoulder Muscle Imbalance as a Risk for Shoulder Injury in Elite Adolescent Swimmers: A Prospective Study
}

\author{
by \\ Joffrey Drigny 1,2,3, Antoine Gauthier, ${ }^{3,4}$ Emmanuel Reboursière1, Henri Guermont ${ }^{1}$, \\ Vincent Gremeaux ${ }^{5,6}$, Pascal Edouard6,7,8
}

\begin{abstract}
Muscle strength imbalances between the internal and external rotators of the shoulder are frequent in swimmers, but their role in shoulder injury remains unknown. We aimed to evaluate the association of shoulder rotator strength and injury in elite adolescent swimmers. Eighteen adolescent swimmers performed preseason isokinetic tests of the internal and external rotator muscles in concentric (con) and eccentric (ecc) modes. Conventional (conER:conIR and eccER:eccIR) and functional ratios (eccER:conIR and eccIR:conER) were calculated. Thirteen swimmers completed a weekly questionnaire about swimming habits and shoulder injuries throughout the season. Preseason testing showed a significant negative association between the functional eccER:conIR ratio and years of practice ( $p<0.05)$. Over the season, $46 \%$ of athletes experienced at least one shoulder injury. At the end of the season, peak torques increased for both internal and external rotator muscles strength, but only concentrically, resulting in a decrease in the eccER:conIR functional ratio ( $p$ $<0.05)$. The receiver operating characteristic curve analysis highlighted good predictive power for the preseason functional eccER:conIR ratio, as values below 0.68 were associated with a $4.5-$ fold (95\% CI 1.33-15.28, $p<0.05)$ increased risk of shoulder injuries during the season.
\end{abstract}

Key words: swimming, sports injury prevention, isokinetic testing, strength imbalance.

\section{Introduction}

Shoulder pain is the most frequent musculoskeletal complaint in swimming, affecting from 40 to $91 \%$ of athletes (Matzkin et al., 2016), and $20-35 \%$ of competitive swimmers experience a time-loss injury each year (Gaunt and Maffulli, 2012). Adolescent swimmers are exposed to an increased risk of injury when transitioning from one to two practices daily with a high training volume (Bak, 2010; Wolf et al., 2009). The high injury rate in swimmers could be due to swimming mechanics consisting of specific repetitive motion. Indeed, swimming propulsion mainly relies on repetitive adduction and medial rotation of the shoulders, often leading to muscular imbalances between strong internal rotators (IR) and relatively weaker external rotators (ER) (Batalha et al., 2013).

Assessing rotator muscle imbalance in swimmers thus appears highly relevant and feasible in clinical settings using isokinetic testing, which is an objective, useful and reliable tool to quantify muscular strength and imbalances

1 - Normandie Univ, UNICAEN, Sports Medicine Unit, CHU de Caen Normandie Caen, France.

2 - Normandie Univ, UNICAEN, Physical Medicine and Rehabilitation Unit, CHU de Caen Normandie Caen, France.

3 - UMR-S 1075 INSERM/Unicaen COMETE, Caen, France.

4 - Université de Caen Basse Normandie, UPRES-EA 2131, UFR STAPS, Caen, France.

5 - Swiss Olympic Medical center, Centre de médecine du sport, Division de médecine physique et réadaptation, Centre Hospitalier Universitaire Vaudois, Lausanne, Switzerland.

6 - Institute of Sport Sciences, University of Lausanne, Lausanne, Switzerland.

7 - Inter-university Laboratory of Human Movement Science (LIBM EA 7424), University of Lyon, University Jean Monnet, F42023. Saint Etienne, France.

8 - Department of Clinical and Exercise Physiology, Sports Medicine Unit, University Hospital of Saint-Etienne, Faculty of Medicine, Saint-Etienne, France.

Authors submitted their contribution to the article to the editorial board.

Accepted for printing in the Journal of Human Kinetics vol. 75/2020 in October 2020. 
(Batalha et al., 2013; Collado-Mateo et al., 2018; Olivier and Daussin, 2018). Strength imbalances are evaluated using the conventional ratio, calculated as the ratio between ER and IR peak torques (PTs) in a concentric (conER:conIR) or eccentric contraction mode (eccER:eccIR). For injury prevention, the ER:IR ratio should be between 0.66 and 0.75 (Ellenbecker and Davies, 2000), and consequently, external rotators must have at least $2 / 3$ of the internal rotator strength. More recently, the functional ratio, which is calculated as the ratio between the ER PT during an eccentric action and the IR PT during a concentric action (eccER:conIR), has been considered to be more appropriate to evaluate the eccentric action of the antagonist muscles, contributing to dynamic glenohumeral joint stability. This ratio has been studied mainly in overhead sports (Andrade et al., 2013; Guney et al., 2016), in which ratios above 1.0 are considered reference values (Andrade et al., 2013). In highlevel front-crawl swimmers, Olivier et al. (2008) showed a significantly lower conventional concentric ratio when compared to nonswimmers (conER:conIR 0.52 vs. 0.75). In adolescent swimmers, Batalha et al. (2013) studied changes in the conventional concentric ratio after 32 weeks of training and reported a significant decrease in conER:conIR from 0.79 to 0.71 . Some studies have shown that athletes with shoulder instability or impingement had a lower functional eccER:conIR ratio (Güney et al., 2014; Saccol et al., 2010) and that it could be the origin of shoulder injuries, in general and in swimmers (Bradley et al., 2016). Moreover, Bak and Magnusson (1997) also reported functional ratios below 1.0 in asymptomatic swimmers (eccER:conIR $=0.86$ ). The other functional work ratio is calculated as eccIR:conER, which reflects the action of arm cocking in overhead throwing, and eccIR:conER $>1.61$ has been described as an injury risk in handball (Edouard et al., 2013).

However, it is unclear whether this muscular imbalance causes shoulder injuries in swimmers (Bradley et al., 2016) or is simply a physiological adaptation to training. In this context, we aimed to evaluate the association between preseason isokinetic shoulder strength testing and shoulder injuries in elite adolescent swimmers. A secondary objective was to characterize the individual variables associated with a muscle imbalance.

\section{Methods}

We conducted a prospective cohort study to investigate the in-season changes of shoulder IR and ER PTs and ratios and the incidence of shoulder injury among adolescent swimmers. This study was conducted in a University Hospital Center. The protocol was approved by an independent ethics committee for research (CERSTAPS, 2016-05-11-16), and the informed consent of each of the participants, or their legal representatives in the case of minors, was obtained. The protocol was conducted according to the Declaration of Helsinki.

\section{Participants}

The study population was all swimmers from an adolescent elite group, defined as nationallevel competitors (in their age categories), training twice daily. Swimmers were included on a voluntary basis. Exclusion criteria were swimmers with a current or recent $(<1$ month) shoulder injury with time lost from sport participation and those unable to perform the isokinetic test. For the prospective follow-up, we excluded swimmers who were unable to participate in the computerized follow-up or those who ended the season for a reason other than a shoulder injury. Among the 25 swimmers in the whole group, 18 participants (10 males, 8 females) were included, and their characteristics are described in Table 1; $39 \%$ reported a shoulder injury in the previous season. They were mainly sprinters $(56 \%$ as defined by their best performance for a less than $200 \mathrm{~m}$-distance) and front-crawl swimmers (44\%). Thirteen participants were followed during the whole season. Their characteristics are reported in Table 1 and did not have significant differences from the whole group $(\mathrm{n}=18)$. Among the 5 swimmers who did not participate, one had a severe lower limb injury (ACL rupture), 2 swimmers pursued their careers abroad, and 2 swimmers did not wish to continue the follow-up.

\section{Measures}

Shoulder isokinetic strength data collection

The shoulder isokinetic strength profile was assessed using a Con-Trex® isokinetic dynamometer (Con-Trex MJ; CMV AG, Dübendorf, Switzerland). The PT (Nm) and range of motion $\left(^{0}\right)$ were recorded. The testing apparatus was set up as described in the owner's manual, and participants were placed in a seated position, with $45^{\circ}$ of shoulder abduction in the scapular plane, 
according to the position described by Davies (1987). The ROM for testing was $70^{\circ}\left(20^{\circ}\right.$ for internal rotation and $50^{\circ}$ for external rotation). All swimmers had a familiarization set of 3 submaximal repetitions under all conditions before recording. Data were collected from the first set of 5 maximal repetitions at $60^{\circ} \cdot \mathrm{s}^{-1}$ in the concentric mode (con) and then the second set of 4 maximal repetitions at $60^{\circ} \cdot \mathrm{s}^{-1}$ in the eccentric mode (ecc), with constant verbal stimulation. The participants' arms were passively weighted to provide gravity compensation data, and corrections were incorporated (Edouard et al., 2009). The studied variables were $\mathrm{PT}$, which is the maximum torque of force normalized to body weight $(\mathrm{Nm} / \mathrm{kg})$, the conventional conER:conIR ratio and eccER:eccIR ratio at $60^{\circ} \cdot \mathrm{s}^{-1}$, and the functional eccentric-toconcentric ratios eccER:conIR and eccIR:conER at $60^{\circ} \cdot \mathrm{s}^{-1}$, for both the dominant and non-dominant sides.

Prospective injury data collection

During the 2016-17 season, all swimmers had to complete a weekly computerized questionnaire. All shoulder injuries were documented (type and duration), including time loss and treatment. A significant shoulder injury was defined as one that interfered with training or progress in training, consistent with the literature about swimming injuries (Gaunt and Maffulli, 2012; Walker et al., 2012). We used the National Collegiate Athletic Association Injury Surveillance System (NCAA-ISS) definition and collected data on injuries that occurred as a result of swimming participation, required medical attention and resulted in significant participation time lost for 1 or more calendar days beyond the day of injury (Dick et al., 2007).

\section{Design and procedures}

The evaluations were carried out twice at a 40-week interval: (1) at the beginning of the competitive season (preseason visit), and (2) at the end of the competitive season (postseason visit). At the preseason visit, all 18 swimmers completed a self-administered questionnaire about their sociodemographic status, training habits, swimming performance and previous injuries. A standardized clinical examination including specific physical examination tests of the shoulder was performed by a sport medicine physician (JD). The shoulder strength profile was assessed by isokinetic testing as described above. The follow- up was carried out over 38 weeks (October 2016 to July 2017). At the postseason visit, all swimmers included in the follow-up had the same evaluations as those at the preseason visit, with the same examiner (JD).

\section{Statistical analyses}

Means and standard deviations (SD) were calculated for all variables. The normal distribution of the data was checked by the Kolmogorov-Smirnov test. A 2-way ANOVA was used to determine the effect of swimmers' characteristics on the preseason PT, ratios and inseason changes. Regression analysis with an F-test and the r-squared $\left(\mathrm{r}^{2}\right)$ statistic was used to determine the effect of age or experience variance on PT or ratio parameters. ANCOVA was used to estimate differences between IR and ER on the inseason changes. To assess the predictive accuracy of muscular imbalances, we used the receiver operating characteristic (ROC) analysis (Hanley and McNeil, 1982). The optimal threshold values (criteria) were associated with a greater Youden index $(\mathrm{J}=$ sensitivity + specificity - 1$)$, and a chisquared test with Yates correction was used to determine the relative risk (RR) of shoulder injury. Statistical analyses were performed with IBM SPSS Statistics for Windows, Version 20.0. (Armonk, NY: IBM Corp.).

\section{Results}

\section{Shoulder strength profile in swimmers $(n=18)$}

The isokinetic PTs and ratios from preseason testing are presented in Table 2. Both conIR and eccIR PTs were significantly greater in male than in female swimmers ( $\mathrm{t}: 2.42-2.90, p<$ $0.05)$. The years of practice explained a significant proportion of variance in the PTs under all conditions (conIR, conER, eccIR and eccER) $\left(\mathrm{r}^{2}=\right.$ $0.40-0.59, p<0.01)$ and in the functional eccER:conIR ratio $\left(\mathrm{r}^{2}=0.25, p<0.05\right)$ (Figure 1$)$.

\section{In-season changes in shoulder strength in swimmers $(n=13)$}

Participants had a mean of $6.7 \pm 1.1$ weekly swimming sessions, corresponding to an average total of $34.3 \pm 5.7 \mathrm{~km}$ per week, and they had $1.5 \pm$ 0.3 weekly dry-land sessions. Swimming sessions were based on elite competitive swimming practices. A standardized resistance training session consisted of a 10-min dynamic warm-up, a pyramidal weight set on the squat, bench press, and pull-down/seal row exercises successively and 
finally a core strengthening exercise routine.

For the 13 swimmers included in the follow-up, in-season changes between preseason and postseason testing for the PT and ratio parameters are presented in Figure 2. The conIR and conER PTs significantly increased $(t=-2.57$ to -3.83, $p<0.05)$ during the season without a difference between IR and ER PTs gains in both the dominant $(\mathrm{F}[2.24]=0.05, p=0.82)$ and nondominant $\operatorname{arm}(\mathrm{F}[2.24]=0.19, p=0.66)$. The conventional conER:conIR and eccER:eccIR ratios did not change significantly during the season. No change was found in the eccentric mode. At the end of the season, both functional ratios showed a significant decrease in eccER:conIR and eccIR:conER ratios compared to those from the preseason $(\mathrm{t}=2.59, p<0.05)$.

\section{Association of rotator strength and shoulder injury}

Among the follow-up group, 6 swimmers
(46\%) sustained at least one shoulder injury during the season. Five swimmers had a rotator cuff tendinopathy (supra-spinatus muscle and long head of the biceps muscle), and one swimmer had a grade-two deltoid injury. The average time loss was $3.8 \pm 2.0$ days. The ROC curves for the PT ratios as predictors of the risk of shoulder injury showed that the eccER:eccIR ratio (ROC-AUC analysis, AUC $=0.79,95 \%$ Confidence Interval [CI]: 0.20$0.96, p<0.05)$ and eccER:conIR ratio (AUC $=0.79$, 95\% CI: 0.29-0.95, $p<0.05$ ) had accurate predictive ability to discriminate injury. After interpretation of the Youden index, the muscular imbalance was defined as eccER:eccIR $<0.66$ (sensitivity $=0.86$, specificity $=0.83 ; \mathrm{J}=0.49$ ) and eccER:conIR $<0.68$ (sensitivity $=1.00$, specificity $=0.67, \mathrm{~J}=0.67)$. The relative risks of shoulder injuries associated with preseason muscle imbalances are presented in Table 3. There was a significant relative risk (RR) of 4.50 (95\% CI: 1.33-15.28, $p<0.05)$ when the participant had eccER:conIR $<0.68$.

\begin{tabular}{|c|c|c|}
\hline \multicolumn{3}{|c|}{$\begin{array}{l}\text { Characteristics of the swimmers in the initial group }(n=18) \\
\text { and swimmers included in the follow-up }(n=13)\end{array}$} \\
\hline & Total $\mathrm{n}=18$ & Follow-up $\mathrm{n}=13$ \\
\hline Age (year \pm SD) & $16.1 \pm 2.3$ & $16.3 \pm 1.7$ \\
\hline Female ( $\mathrm{n}$ and $\%$ ) & $8(44 \%)$ & $6(46 \%)$ \\
\hline Body weight $(\mathrm{kg} \pm \mathrm{SD})$ & $62.1 \pm 12.8$ & $58.3 \pm 10.0$ \\
\hline Height $(\mathrm{cm} \pm \mathrm{SD})$ & $172.5 \pm 11.3$ & $169.23 \pm 10.0$ \\
\hline $\mathrm{BMI}\left(\mathrm{kg} / \mathrm{m}^{2} \pm \mathrm{SD}\right)$ & $20.6 \pm 2.1$ & $20.20 \pm 1.9$ \\
\hline Right dominant arm (n and \%) & $17(94 \%)$ & $12(92 \%)$ \\
\hline Career lengths (year $\pm S D$ ) & $8.2 \pm 2.9$ & $7.4 \pm 2.4$ \\
\hline Front-crawl specialist (n and \%) & $8(44 \%)$ & $6(46 \%)$ \\
\hline Symmetric stroke (n and \%) & $5(28 \%)$ & $3(23 \%)$ \\
\hline Sprint $<200 \mathrm{~m}(\mathrm{n}$ and $\%)$ & $10(56 \%)$ & $7(53 \%)$ \\
\hline Swimming performance (FINA pts \pm SD) & $574.3 \pm 79.4$ & $564.92 \pm 84.3$ \\
\hline Previous year shoulder injury & $7(39 \%)$ & $5(38.5 \%)$ \\
\hline \multicolumn{3}{|c|}{$\begin{array}{l}\text { SD: standard deviation; BMI: body mass index; FINA: Fédération Internationale de Natation } \\
\text { No significant differences were found between the total and follow-up groups. }\end{array}$} \\
\hline
\end{tabular}


Table 2

Isokinetic variables of the peak torques and the ratios at the preseason visit with respect to sex

Total $n=18 \quad$ Male $n=10 \quad$ Female $n=8$

\begin{tabular}{lllllll} 
& Dom & Non-dom & Dom & Non-dom & Dom & Non-dom \\
\hline Peak Torque $(\mathrm{Nm} / \mathrm{kg})$ & & & & & & \\
conIR at $60^{\circ} \cdot \mathrm{s}^{-1}$ & $0.73 \pm 0.24$ & $0.69 \pm 0.21$ & $0.85 \pm 0.28$ & $0.80 \pm 0.21$ & $0.59 \pm 0.10^{\text {a }}$ & $0.55 \pm 0.12$ \\
conER at $60^{\circ} \cdot \mathrm{s}^{-1}$ & $0.47 \pm 0.13$ & $0.45 \pm 0.11$ & $0.51 \pm 0.14$ & $0.50 \pm 0.17$ & $0.41 \pm 0.10$ & $0.39 \pm 0.06$ \\
eccIR at $60^{\circ} \cdot \mathrm{s}^{-1}$ & $0.81 \pm 0.25$ & $0.74 \pm 0.2$ & $0.96 \pm 0.24$ & $0.85 \pm 0.2$ & $0.63 \pm 0.06^{\text {a }}$ & $0.62 \pm 0.08$ \\
eccER at $60^{\circ} \cdot \mathrm{s}^{-1}$ & $0.51 \pm 0.12$ & $0.50 \pm 0.15$ & $0.56 \pm 0.13$ & $0.56 \pm 0.18$ & $0.46 \pm 0.06$ & $0.44 \pm 0.08$ \\
\hline Ratio $\left(\right.$ at $60^{\circ} \cdot \mathrm{s}^{-1}$ ) & & & & & & \\
Conventional conER:conIR & $0.65 \pm 0.11$ & $0.68 \pm 0.13$ & $0.62 \pm 0.09$ & $0.62 \pm 0.05$ & $0.70 \pm 0.12$ & $0.74 \pm 0.18$ \\
Conventional eccER:eccIR & $0.65 \pm 0.11$ & $0.68 \pm 0.11$ & $0.59 \pm 0.09$ & $0.62 \pm 0.09$ & $0.73 \pm 0.08$ & $0.71 \pm 0.12$ \\
Functional eccER:conIR & $0.72 \pm 0.11$ & $0.75 \pm 0.18$ & $0.70 \pm 0.11$ & $0.69 \pm 0.11$ & $0.78 \pm 0.07$ & $0.83 \pm 0.22$ \\
Functional eccIR:conER & $1.76 \pm 0.37$ & $1.65 \pm 0.24$ & $1.86 \pm 0.38$ & $1.69 \pm 0.23$ & $1.59 \pm 0.31$ & $1.59 \pm 0.26$ \\
\hline
\end{tabular}

a: Significant difference between males and females ( $p$-value $<0.05$ )

ER: external rotation, IR internal rotation, con: concentric, ecc: eccentric,

Dom: dominant, Non-dom: non-dominant

Table 3

The relative risk of swimmer shoulder injury associated with muscular imbalances $(n=13)$

\begin{tabular}{|c|c|c|c|c|}
\hline $\begin{array}{l}\text { Accuracy } \\
\text { (AUC-ROC) }\end{array}$ & $p$-value & Criteria & Relative Risk & $\begin{array}{l}\text { Confidence } \\
\text { Interval (95\%) }\end{array}$ \\
\hline
\end{tabular}

Conventional ratios

\begin{tabular}{|c|c|c|c|c|c|}
\hline conER:conIR at $60 \% \mathrm{~s}$ & 0.64 & 0.20 & $<0.66$ & 1.71 & 0.47 to 6.30 \\
\hline eccER:eccIR at $60 \% \mathrm{~s}$ & $0.79^{a}$ & 0.04 & $<0.66$ & 5.83 & 0.92 to 37.08 \\
\hline \multicolumn{6}{|l|}{ Functional ratios } \\
\hline eccER:conIR at $60 \% \mathrm{~s}$ & $0.79^{a}$ & 0.03 & $<0.68$ & $4.50^{\mathrm{b}}$ & 1.33 to 15.28 \\
\hline eccIR:conER at $60 \% \mathrm{~s}$ & 0.69 & 0.26 & $>1.37$ & 3.75 & 0.38 to 36.76 \\
\hline & & & $\geq 2$ criteria & 3.20 & 0.89 to 11.48 \\
\hline
\end{tabular}

Global muscular imbalance

a Significant accuracy with p-value $<0.05$ for tests of the null hypothesis that the AUC-ROC equals 0.50

b Significant relative risk when the confidence interval (95\%) was above and did not contain the value 1 ( $p$-value < 0.05). AUC: area under the curve; ROC: receiver operating characteristic;

ER: external rotation; IR: internal rotation; ecc: eccentric; con: concentric 


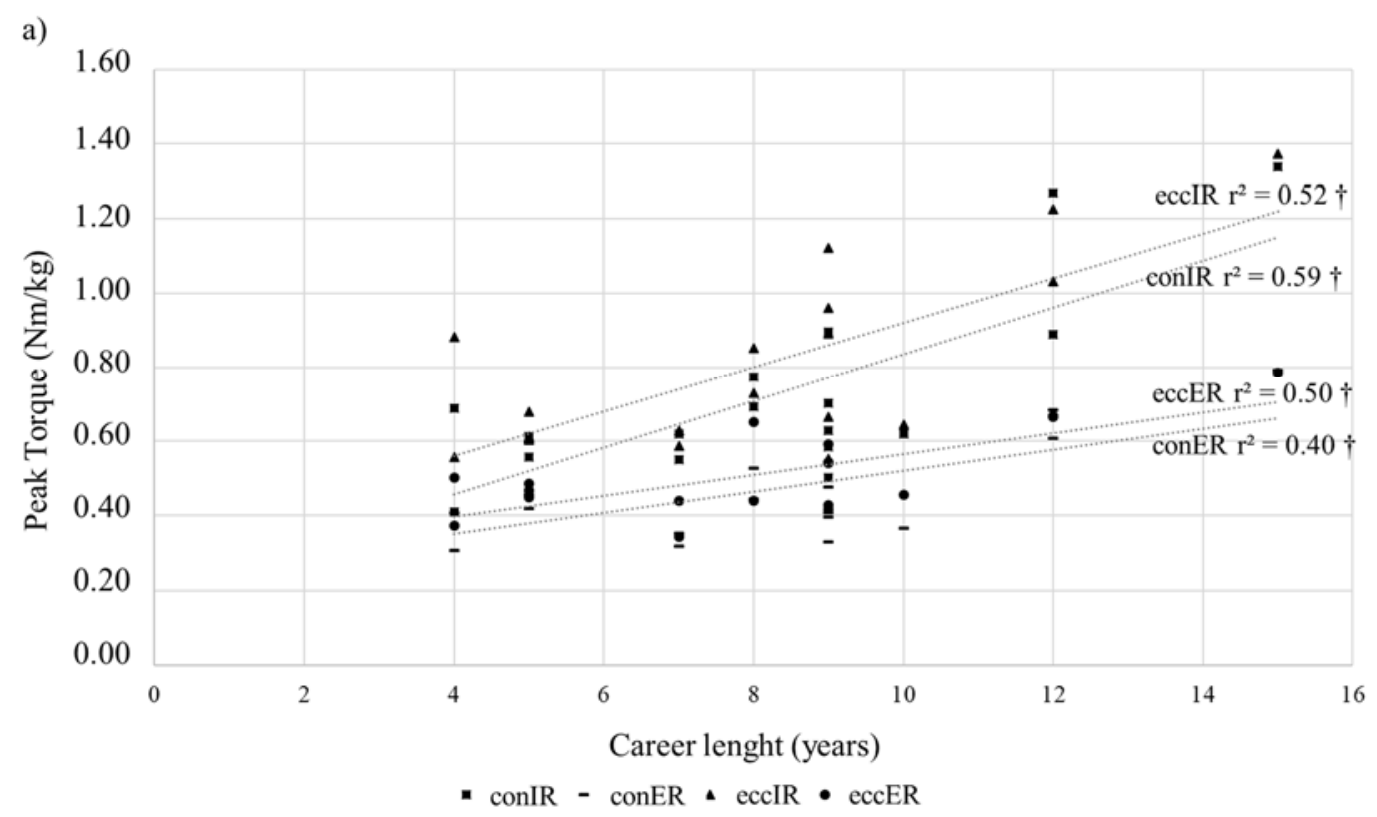

b)

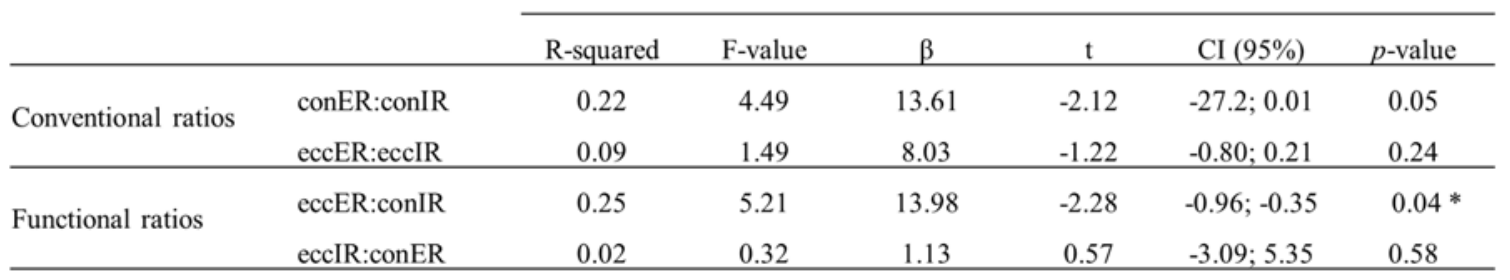

\section{Figure 1}

Isokinetic peak torques (a) at the preseason visit with respect to the swimming career length in years and $(b)$ the regression analysis of the ratios with respect to the swimming career length in years $(n=18)$ con : concentric, ecc : eccentric, ER: external rotators, IR: internal rotators

$\beta$ : regression beta coefficients, $t: \beta$ associated $t$-value, CI: confidence interval; ${ }^{*} p$-value $<0.05+p$-value $<0.01$ 
a)

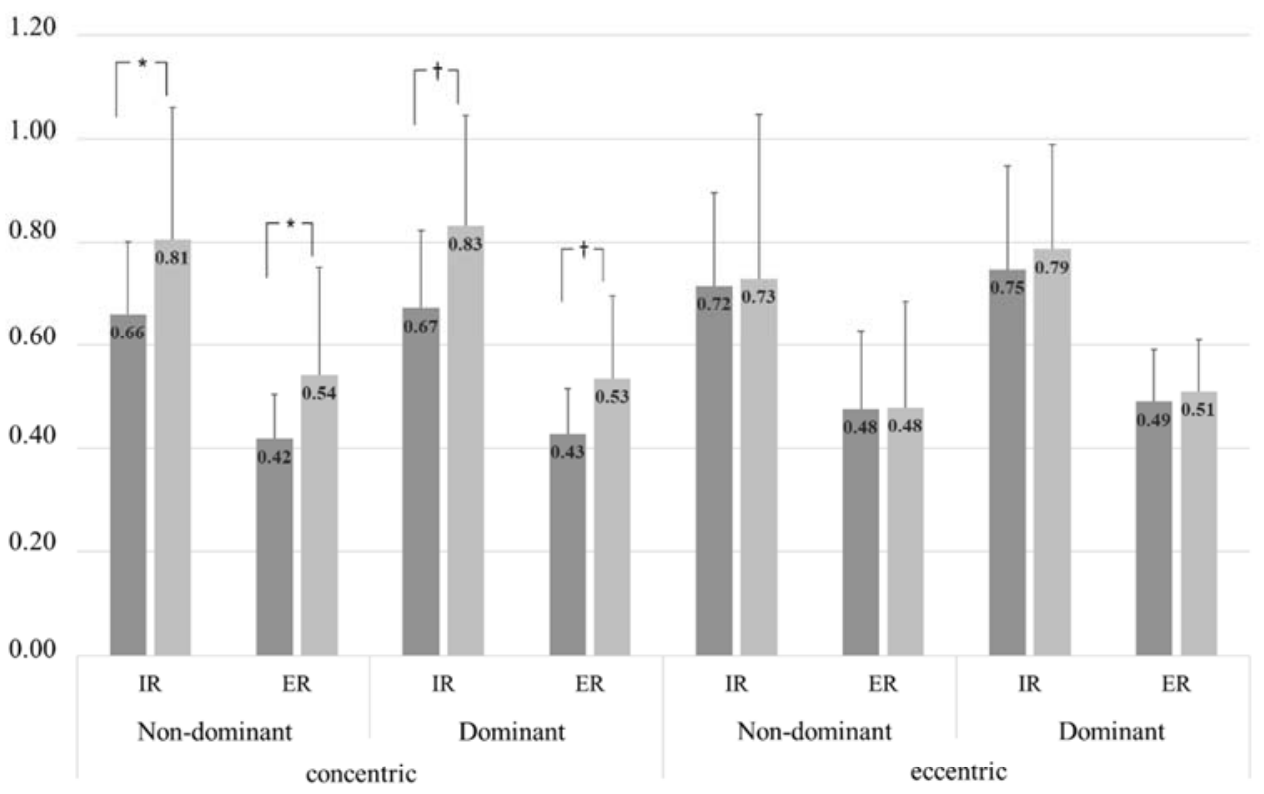

b)

2.50

2.00

1.50

1.00

0.50
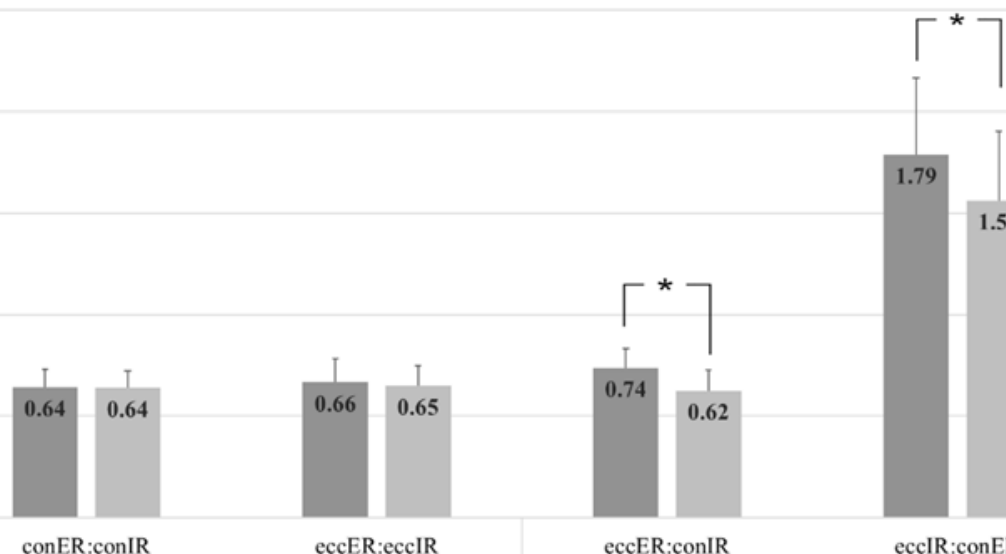

0.00

conER:conIR

eccER:conIR

eccIR:conER

Conventional Ratio

Dominant

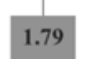

= preseason $=$ postseason

Figure 2

Changes in in-season isokinetic peak torques (a) and ratios $(b)$ in swimmers $(n=13)$ con : concentric, ecc : eccentric, ER: external rotators, IR: internal rotators ${ }^{*}$ p-value $<0.05+p$-value $<0.01$ 


\section{Discussion}

In our cohort, swimmers with a lower preseason conventional eccentric eccER:eccIR ratio and functional eccER:conIR ratio had an increased risk of shoulder injury during the season. A preseason functional eccER:conIR ratio below 0.68 was associated with a significant 4.5 -fold increased risk of developing a shoulder injury $(R R=4.50 \mathrm{p}<$ 0.05).

The functional eccER:conIR ratio may be an interesting and relevant variable for identifying swimmers at higher risk for shoulder injury (with a cut-off at 0.68 in our study). Previous authors also conducted prospective studies and evaluated the associations of preseason shoulder rotator isokinetic measurements and injuries in overhead athletes (Edouard et al., 2013; Wang et al., 2000). Lower eccER:conIR functional ratios have also been described as injury risk factors in elite volleyball players (Wang et al., 2000) or handball players (Edouard et al., 2013). It is also interesting to note that the criterion of a functional eccER:conIR ratio $<0.68$ is very close to that adopted in the study of Edouard et al. (2013), in which a threshold ratio of 0.67 was calculated from the study of a control, non-athlete population. Moreover, the conventional eccentric eccER:eccIR ratio had the predictive ability to identify swimmers at risk for shoulder injury, but the cutoff $(<0.66)$ failed to show statistically significant results and was thus unlikely to be clinically relevant and applicable in clinical practice.

Until now, the literature on rotator muscle imbalance and its association with shoulder injury in swimmers was mainly based on conventional concentric ratios (Bak, 2010; Batalha et al., 2013; Bradley et al., 2016). However, external rotators are less likely to be involved in their concentric mode as external rotation is the least used motion in swimming and is mostly involved in the nonpropulsive phase (Manske et al., 2015). Thus, the main function of the ER muscles in the propulsion phase is to limit the anterior translation of the humeral head by contracting eccentrically to counteract the propulsive internal rotation (Gaudet et al., 2018). Electromyographic studies on swimming strokes found the highest activity of external rotator muscles (teres minor) during the pull-through phase (Pink et al., 1993) and consequently, when operating in an eccentric mode. The high eccentric demands on the ER muscles have been implicated in the impairment of control of glenohumeral-joint translation, ultimately predisposing the shoulder to injury (Bradley et al., 2016; Ramsi et al., 2004), and an analysis of muscular recruitment in swimmers demonstrated a decrease and a delay in the activation of $\mathrm{m}$. infraspinatus in swimmers with impingement syndrome (Scovazzo et al., 1991). However, to our knowledge, this is the first study to prospectively analyze the predictive impact of the muscle imbalance on the risk of shoulder injury in swimmers.

Moreover, our results highlight that the functional eccER:conIR and eccIR:conER ratios decreased during the season, mainly due to the increase in concentric strength of both internal and external rotators, which was not associated with a similar increase in eccentric strength. This can be interpreted as a worsening of the functional eccER:conIR ratio, with post-season mean values $(0.62 \pm 0.10)$ that were lower than the preseason cutoff found to indicate the risk of injury (0.68). This suggests that high-level swimming training induces insufficient eccentric strengthening compared to concentric strengthening that may expose the shoulder to instability and injury (Gaudet et al., 2018), which worsen during the competitive season. On the other hand, the conventional conER:conIR and eccER:eccIR ratios did not significantly change during the season. These results are not consistent with the finding from Batalha et al. (2013) who found a decrease in the conER:conIR ratio from 0.79 to 0.71 during a competitive season in adolescent swimmers. However, in our sample, swimmers already had lower conventional ratios in the preseason tests $(0.65 \pm 0.11)$.

Both IR and ER PTs were positively associated with age in all modes (ecc and con) and highly related to sport participation, as the normalized PT was stable in the control population between 13 and 36 years (Andrade et al., 2013). We also confirmed that swimmers are exposed to muscular imbalances, with a mean conventional conER:conIR ratio of 0.65 and a mean functional eccER:conIR ratio of 0.72 . The literature supports that conventional conER:conIR ratio values below 0.66 characterize muscle imbalances which increase the risk for injury or instability (Ellenbecker and Davies, 2000) and that functional 
eccER:conIR ratios above 1.0 should be targeted in swimmers (Bradley et al., 2016).

The present study shows that there was a significant negative correlation between the years of practice and the functional eccER:conIR ratio and a statistical trend toward significance for the conventional conER:conIR ratio. This suggests that shoulder rotator muscle imbalances may worsen as the years of practice increase and could increase the risk of shoulder injury over time (in addition to age per se). As shoulder pain increases with the years of practice (Dischler et al., 2018), we hypothesized that a specific prevention program aiming to reduce muscle imbalance could be targeted at young swimmers undergoing highvolume training (Batalha et al., 2018). Further studies with a long-term follow-up are needed to confirm these hypotheses. Moreover, although isokinetic evaluation is not currently a standard evaluation in swimming, it is the first step in optimizing the follow-up of swimmers. In swimmers, especially those with muscular imbalances, a specific land-based eccentric program targeting the external rotators (Tessaro et al., 2017) could limit the risk of injury.

These results are presented with caution as this is a preliminary trial of this approach, and there are several limitations to this study. First, the number of participants included in the current study was limited, and further studies are needed to confirm the results for larger samples. Second, this is a monocentric study, as we included swimmers from a single swimming club, but the swimmers' characteristics and training habits were consistent with national trends for elite adolescent swimmers. Thirdly, functional ratios were calculated with successive sets of either concentric or eccentric internal/external rotations tested independently. In the literature, protocols are heterogenous with some studies using concentric force consecutively followed by the eccentric force on the same set. Further studies are needed to compare and evaluate both protocols. Additionally, while injuries have been confirmed by health professionals, swimmers did not benefit from systematic imaging. However, all injuries were common overuse injuries in swimmers, for which diagnostic criteria are largely based on clinical variables. Moreover, a recent study described high prevalence of imaging abnormalities in the shoulders of swimmers, even in asymptomatic athletes (Rodeo et al., 2016). Finally, some teams described isokinetic evaluations in other positions when testing shoulder rotation (sitting, standing, or supine). However, Davies' seated position has high reliability and good clinical practical implications (Edouard et al., 2011).

This study reports that preseason functional eccER:conIR ratios were associated with a higher risk for developing shoulder injuries in elite adolescent swimmers. A functional eccER:conIR ratio below 0.68 identified swimmers with a 4.5-fold increased risk of in-season shoulder injury. In addition, our results highlighted that high-level swimming practice induced a significant decrease in the functional eccER:conIR ratio during a competitive season, which could lead to an increased risk of shoulder injury over time. Further studies on larger populations are needed to confirm these results, identify populations at risk and assess the efficacy of specific strengthening programs in reducing muscle imbalances to decrease the incidence of swimming-related shoulder injuries.

\section{References}

Andrade MS, Vancini RL, de Lira CAB, Mascarin NC, Fachina RJFG, da Silva AC. Shoulder isokinetic profile of male handball players of the Brazilian national team. Braz J Phys Ther, 2013; 17: 572-578

Bak K. The practical management of swimmer's painful shoulder: etiology, diagnosis, and treatment. Clin J Sport Med, 2010; 20: 386-390

Bak K, Magnusson SP. Shoulder strength and range of motion in symptomatic and pain-free elite swimmers. Am J Sports Med, 1997; 25: 454-459

Batalha N, Dias S, Marinho DA, Parraca JA. The effectiveness of land and water based resistance training on shoulder rotator cuff strength and balance of youth swimmers. J Hum Kinet, 2018; 62: 91-102 
Batalha NM, Raimundo AM, Tomas-Carus P, Barbosa TM, Silva AJ. Shoulder rotator cuff balance, strength, and endurance in young swimmers during a competitive season. J Strength Cond Res, 2013; 27: 25622568

Bradley J, Kerr S, Bowmaker D, Gomez J-F. Review of shoulder injuries and shoulder problems in competitive swimmers. Am J Sports Sci Med, 2016; 4: 57-73

Collado-Mateo D, Dominguez-Muñoz FJ, Batalha N, Parraça J, Tomas-Carus P, Adsuar JC. Test-retest reliability of isokinetic arm strength measurements in competitive swimmers. J Hum Kinet, 2018; 65: $5-11$

Davies GJ. A compendium of isokinetics in clinical usage and rehabilitation techniques. Onalaska: S \& S Publishers; 1987

Dick R, Agel J, Marshall SW. National collegiate athletic association injury surveillance system commentaries: introduction and methods. J Athl Train, 2007; 42: 173-182

Dischler JD, Baumer TG, Finkelstein E, Siegal DS, Bey MJ. Association between years of competition and shoulder function in collegiate swimmers. Sports Health, 2018; 10: 113-118

Edouard P, Calmels P, Degache F. The effect of gravitational correction on shoulder internal and external rotation strength. Isokinet Exerc Sci, 2009; 17: 35-39

Edouard P, Degache F, Oullion R, Plessis JY, Gleizes-Cervera S, Calmels P. Shoulder strength imbalances as injury risk in handball. Int J Sports Med, 2013; 34: 654-660

Edouard P, Samozino P, Julia M, Gleizes Cervera S, Vanbiervliet W, Calmels P, Gremeaux V. Reliability of isokinetic assessment of shoulder-rotator strength: a systematic review of the effect of position. J Sport Rehabil, 2011; 20: 367-383

Ellenbecker TS, Davies GJ. The application of isokinetics in testing and rehabilitation of the shoulder complex. J Athl Train, 2000; 35: 338-350

Gaudet S, Tremblay J, Begon M. Muscle recruitment patterns of the subscapularis, serratus anterior and other shoulder girdle muscles during isokinetic internal and external rotations. J Sports Sci, 2018; 36: 985-993

Gaunt T, Maffulli N. Soothing suffering swimmers: a systematic review of the epidemiology, diagnosis, treatment and rehabilitation of musculoskeletal injuries in competitive swimmers. Br Med Bull, 2012; 103: $45-88$

Guney H, Harput G, Colakoglu F, Baltaci G. The effect of glenohumeral internal-rotation deficit on functional rotator-strength ratio in adolescent overhead athletes. J Sport Rehabil, 2016; 25: 52-57

Güney H, Harput G, Çolakoğlu F, Baltacı G. Functional strength ratio in athletes with and without glenohumeral internal rotation deficit (GIRD). Orthop J Sports Med, 2014; 2: 2325967114S2325900280

Hanley JA, McNeil BJ. The meaning and use of the area under a receiver operating characteristic (ROC) curve. Radiology, 1982; 143: 29-36

Manske RC, Lewis S, Wolff S, Smith B. Effects of a dry-land strengthening program in competitive adolescent swimmers. Int J Sports Phys Ther, 2015; 10: 858-867

Matzkin E, Suslavich K, Wes D. Swimmer's shoulder: painful shoulder in the competitive swimmer. J Am Acad Orthop Surg, 2016; 24: 527-536

Olivier N, Daussin FN. Relationships between isokinetic shoulder evaluation and fitness characteristics of elite french female water-polo players. J Hum Kinet, 2018; 64: 5-11

Olivier N, Quintin G, Rogez J. The high level swimmer articular shoulder complex. Ann Readapt Med Phys, 2008; 51: 342-347

Pink M, Jobe FW, Perry J, Kerrigan J, Browne A, Scovazzo ML. The normal shoulder during the butterfly swim stroke. An electromyographic and cinematographic analysis of twelve muscles. Clin Orthop Relat Res, 1993: 48-59

Ramsi M, Swanik K, Straub S, Mattacola C. Shoulder-rotator strength of high school swimmers over the course of a competitive season. J Sport Rehabil, 2004; 13: 9-18

Rodeo SA, Nguyen JT, Cavanaugh JT, Patel Y, Adler RS. Clinical and ultrasonographic evaluations of the shoulders of elite swimmers. Am J Sports Med, 2016; 44: 3214-3221

Saccol MF, Gracitelli GC, da Silva RT, Laurino CF, Fleury AM, Mdos SA, da Silva AC. Shoulder functional ratio in elite junior tennis players. Phys Ther Sport, 2010; 11: 8-11 
Scovazzo ML, Browne A, Pink M, Jobe FW, Kerrigan J. The painful shoulder during freestyle swimming. An electromyographic cinematographic analysis of twelve muscles. Am J Sports Med, 1991; 19: 577-582

Tessaro M, Granzotto G, Poser A, Plebani G, Rossi A. Shoulder pain in competitive teenage swimmers and it's prevention: a retrospective epidemiological cross sectional study of prevalence. Int J Sports Phys Ther, 2017; 12: 798-811

Walker H, Gabbe B, Wajswelner H, Blanch P, Bennell K. Shoulder pain in swimmers: a 12-month prospective cohort study of incidence and risk factors. Phys Ther Sport, 2012; 13: 243-249

Wang HK, Macfarlane A, Cochrane T. Isokinetic performance and shoulder mobility in elite volleyball athletes from the United Kingdom. Br J Sports Med, 2000; 34: 39-43

Wolf BR, Ebinger AE, Lawler MP, Britton CL. Injury patterns in division I collegiate swimming. Am J Sports Med, 2009; 37: 2037-2042

\section{Corresponding author:}

\section{Dr Joffrey DRIGNY}

Médecine Physique et de Réadaptation,

CHU de Caen, av. de la Côte de Nacre, 14000, Caen,

France

Tel: +3302310647 21,

Fax: +330231065047

E-mail: j.drigny@gmail.com 